\title{
Entrelacs
}

Cinéma et audiovisuel

\section{Machines musicales, machines a faire peur}

Ostinato et note-pédale

\section{Bernard Arbus}

\section{(2) OpenEdition}

Journals

Édition électronique

URL : http://journals.openedition.org/entrelacs/155

DOI : 10.4000/entrelacs. 155

ISSN : 2261-5482

Éditeur

Éditions Téraèdre

Édition imprimée

Date de publication : 1 novembre 2005

Pagination : 97-102

ISBN : 978-2-912868-70-1

ISSN : 1266-7188

Référence électronique

Bernard Arbus, « Machines musicales, machines a faire peur », Entrelacs [En ligne], 5 | 2005, mis en ligne le 01 août 2012, consulté le 19 avril 2019. URL : http://journals.openedition.org/entrelacs/155 ; DOI : 10.4000/entrelacs. 155

Ce document a été généré automatiquement le 19 avril 2019.

Tous droits réservés 


\title{
Machines musicales, machines a faire peur
}

Ostinato et note-pédale

\author{
Bernard Arbus
}

1 La recherche d'éléments musicaux susceptibles de générer de la peur n'est pas neuve. Il existe en effet dans l'histoire de l'opéra tout un répertoire de ces «figuralismes de la terreur : excitation rythmique, répliques hachées, montées subites de la voix dans l'aigu, voltes-face harmoniques, sémantique de la septième diminuée protéiforme, bruitisme symphonique $»^{1}$. Un certain nombre de ces figures sonores se sont figées dans une signification extramusicale, notamment quand la musique cohabite avec des images (opéra ou cinéma) ou des paroles (mélodies, chansons) qui définissent et orientent son pouvoir symbolique.

2 Le film d'horreur ou d'angoisse, pour la violence des émotions qu'il fait naître et parce qu'il utilise pour cela tous les moyens dont il peut disposer, musicaux notamment, ainsi que pour la codification extrême du genre, est un support propice à l'étude de ces éléments musicaux devenus "machines à faire peur " et même, dans le pire des cas, véritables clichés sonores.

3 Les ostinatos et les notes pédales irriguent ainsi la plupart des bandes originales du genre, souvent de manière très efficace en servant parfaitement les propos de l'image.

\section{L'ostinato}

4 L'ostinato est un procédé d'écriture qui consiste à répéter obstinément un motif ou une cellule musicale, à dominance rythmique ou mélodique. Le temps induit par cette répétition est un temps statique : «si (...) la répétition l'emporte sur la différence, l'évolution du discours se gèle peu à peu en statisme $»^{2}$. Associée à des images qui laissent presque toujours présager de l'imminence d'un événement terrifiant, ce temps statique - mais non immobile - scande le mouvement inexorable de la catastrophe et en circonscrit la durée : l'effet cumulatif de la répétition des motifs s'accompagne d'un accroissement de la tension. 
Dans Halloween (film et musique de John Carpenter), un court motif de sept notes (ex. 1) constitue la matrice de l'ostinato et atteste de la présence, plutôt pressentie qu'effective, du tueur :

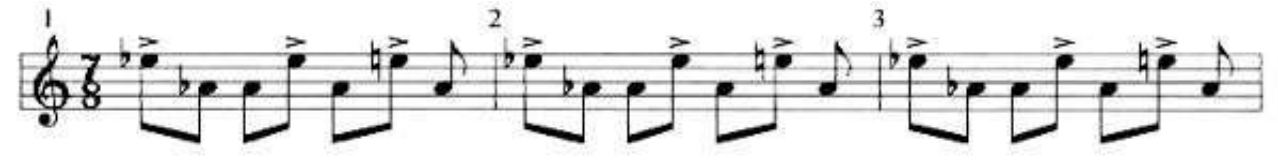

6 Ici, l'ostinato fonctionne à la fois comme signal de reconnaissance, en faisant jouer la mémoire associative qui construit ses propres émotions et comme principe organisateur du mouvement des sujets, menaçant et menacé. La scansion de la formule ne ménage aucune surprise et sert de référentiel rythmique à une action qui devient alors inévitablement programmée et prévisible dans son déroulement ; l'ostinato est vécu avec un sentiment d'inexorabilité vide de toute inflexion humaine.

7 Une technique comparable est à l'œuvre dans Jaws (réalisé par Steven Spielberg, musique de John Williams), où chaque survenue du requin est précédée du célèbre motif chromatique court et nerveux, constitué de deux cellules $\alpha \_$et $\beta$ _de 2 et 4 notes scandées en jeu détaché aux cordes graves (ex. 2), dont la nature élémentaire et quasi archétypale conduit très efficacement la progression du monstre :

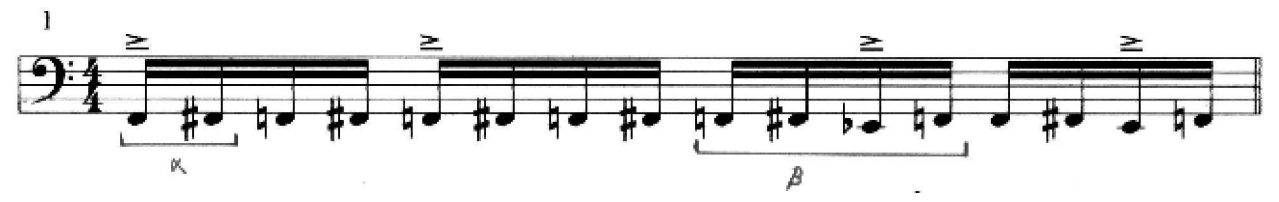

8 Les effets sont en outre renforcés par une accélération et un crescendo d'intensité qui engendrent une tension musicale presque insoutenable, nécessitant une résolution rapide, explosive, à la manière de la strette d'une fugue baroque.

ces exemples, le paradigme de l'attente se construit sur une musique sans devenir n'informant pas, dont le degré de prévisibilité est maximal et où les possibilités de choix s'amenuisent à mesure que les motifs s'accumulent; associée à des séquences cinématographiques qui évoluent en revanche inexorablement et débouchent sur un climax visuel presque toujours sanglant, elle ajoute à l'image, par simple effet dialectique, une forte charge émotionnelle que celle-ci n'aurait pu suffire à produire par sa seule énergie. A la sur-présentation d'un motif musical enfermé dans son dessin initial correspond la représentation d'un personnage qui ne peut échapper à son destin.

Dans un autre film, Le Fantôme de l'opéra (version de Dario Argento, musique de Ennio Morricone), on retrouve la cellule élémentaire de deux notes (ex. 3) en ostinato énoncée lentement -lors de la séquence particulièrement angoissante de la quête d'un hypothétique trésor du fantôme par Alfred et Paulette dans les égouts- qui inscrit dans un temps suspendu le cheminement presque bonhomme des deux personnages :

11 La tension qui s'installe progressivement dans cette séquence sera essentiellement due à la densification de la figure centrale du module $\alpha$ au moyen d'éléments potentiellement instables: notes pédales dynamiques, échos du motif à différents registres, bruits. L'obsessionnelle litanie ne cessera qu'avec la mort d'Alfred (empalé sur une stalagmite), moment où débute le second versant de la séquence consacré à la fuite de Paulette poursuivie par le fantôme, renversement de la situation précédente. Le module musical initial $\alpha_{-}$sera alors relayé par un autre issu du premier selon le procédé du mouvement contraire (ou renversement), toujours en ostinato, mais dans un tempo plus vif : 


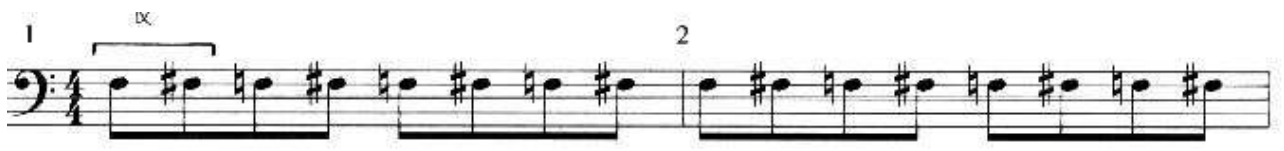

12 Cet exemple montre comment, à partir d'un matériau élémentaire et au moyen d'un ostinato comme principe général d'organisation du discours et de la texture sonore, le compositeur parvient à élaborer une forme musicale bipartite relativement longue (5 minutes environ) dans laquelle la tension croit presque uniformément du début à la fin, répondant parfaitement en cela aux nécessités dramatiques de la séquence cinématographique.

13 L'ostinato produit donc de la tension par accumulation, par le fait d'une répétition envahissante, qui supprime la part de nouveau, suscitant chez l'auditeur une sensation d'enfermement. Par ailleurs, dans les œuvres ci-dessus, ce temps obsessionnel semble vécu comme métaphore du temps de l'agonie, temps désespérément immobile qui prive la mémoire d'objet et donc l'élimine ; et l'homme sans mémoire n'a plus ni passé ni avenir, seulement un présent angoissant, proche de la déterritorialisation schizophrénique ${ }^{3}$, un présent qui n'a que la mort pour limite.

\section{La note-pédale}

Autre procédé d'écriture, autre machine sonore, la note-pédale se retrouve également très souvent dans la musique du film d'épouvante. Techniquement, il s'agit d'une note longuement tenue, pendant que d'autres parties évoluent librement. Cette note peut apparaître dans toute l'étendue des hauteurs, mais dans le genre qui nous intéresse, elle se situera de préférence dans l'infra grave ou le supra aigu. On y retrouve la dimension obsessionnelle que l'on avait pu ressentir dans l'ostinato, mais cette dimension prend un caractère plus intrusif, plus pénétrant qu'envahissant : là où il $\mathrm{y}$ avait dialectique entre le statisme apparent de l'énoncé musical et l'inexorable motricité de l'enchaînement du drame sur l'écran, là où l'ostinato induisait une présence en marche, la pédale définit plutôt un espace, occupé et contaminé par une présence latente, tapie. La note tenue est un témoin, un observateur non encore agissant, mais déjà omniprésent : elle participe du regard plus que du geste.

Dans Vendredi 13(film de Sean. s. Cunnigham, musique de Harry Manfredini), la note pédale révèle la présence de la tueuse cachée dans un placard, pendant que la jeune monitrice et future victime procède à ses ablutions : non seulement elle fonctionne alors comme un véritable signal, dont elle assume le statut - fonction de reconnaissance - et la forme - élémentaire - à la manière simplifiée d'un leitmotiv Wagnérien, mais aussi agit comme relais entre le regard du spectateur et celui du meurtrier, en permettant au premier de "perce-voir » et de " pré-voir » dans un temps arrêté l'évènement à venir tel que l'envisage le second. Car à la différence de l'ostinato, la note pédale s'inscrit dans un temps lisse, non pulsé et d'après Francis Decarsin, «l'utilisation de sons continus, de plages de durée extrêmement distendues, rejoint la notion de temps immobile plutôt que celle d'absence de temps, davantage liée à une répétition trop envahissante $»^{4}$. Ce temps immobile devient alors, par la force de l'image, temps figé en une extase de l'horreur: l'ostinato précipite et enferme, la note pédale suspend et dévoile. 


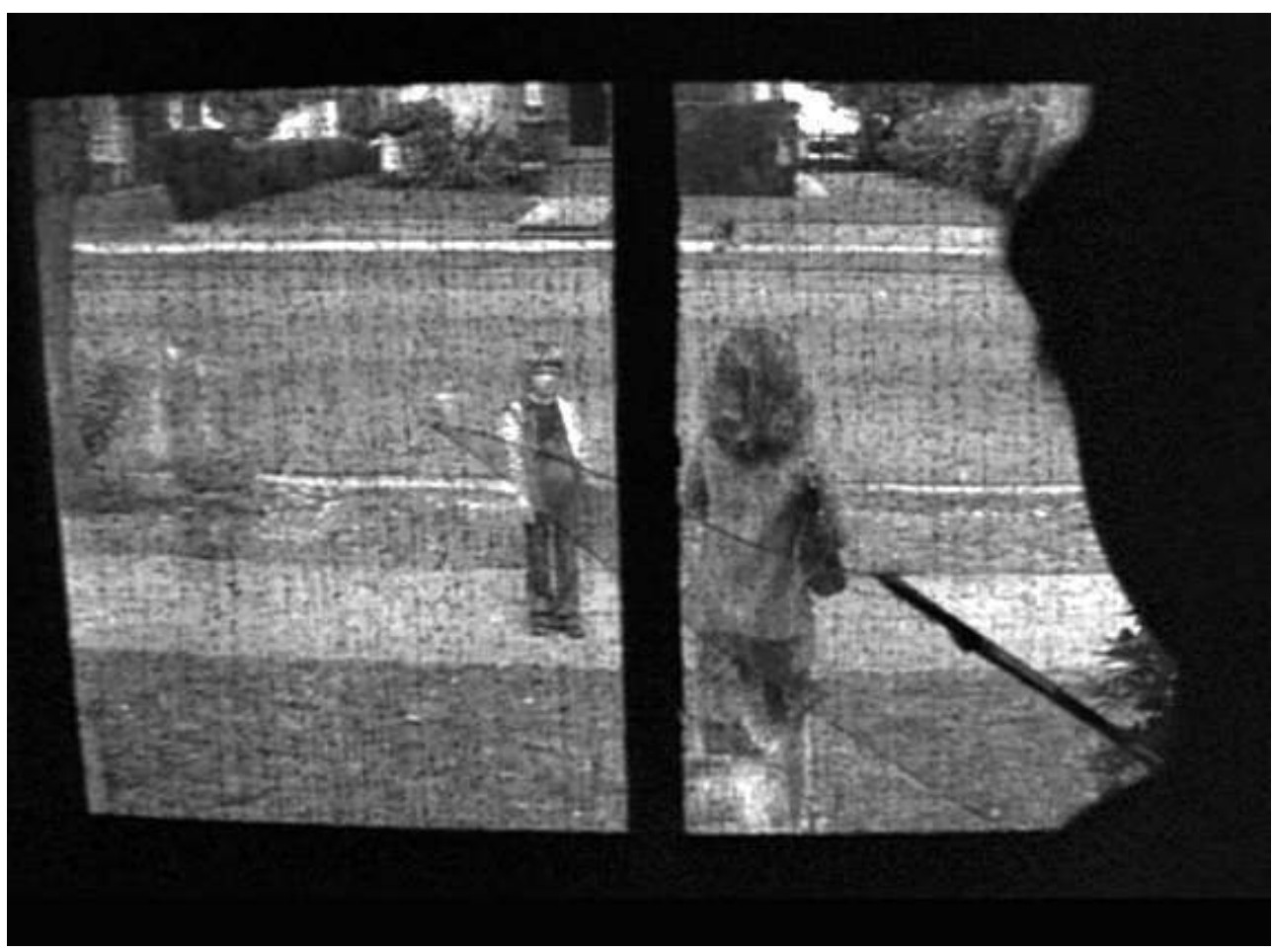

16 Ce procédé est d'ailleurs rarement utilisé dans sa forme pure, à savoir avec une unique note tenue. Souvent vient s'ajouter une autre pédale, située à l'autre extrémité du champ des hauteurs et en contrepoint de la première. La dissonance s'exerce alors dans l'espace à deux niveaux, celui des registres et celui des hauteurs, même si celles-ci restent indiscernables précisément.

17 Cette double opposition vient compléter l'effet de tension déjà induit par la première pédale.

18 Ce procédé est très largement utilisé dans Scream(réalisé par Wes Craven, musique de Marco Beltrami), film dont la musique quasi ininterrompue est au demeurant d'une grande plasticité, réellement plaquée à l'image et en épousant tous les micros et macrosévènements. Passant sans cesse de la description à la représentation, jouant à merveille sur les rapports cinétiques et spatiaux entre le son et les images, elle offre un exemple complet des procédés d'écriture musicale en usage dans le genre, traités avec beaucoup d'efficacité.

19 Ainsi, quand Sydney, en communication téléphonique avec l'un des deux tueurs tente de le localiser autour de sa maison, naît dans les profondeurs du registre grave une sombre pédale très lentement pulsée, à laquelle vont bientôt se superposer des nappes aiguës évoluant faiblement par degrés chromatiques et que viendront enfin animer des pizzicati de cordes en ostinato (ex. 5). Trois qualités de temps sont ainsi successivement vécues par l'audio-spectateur :

1. le temps immobile de la pédale qui manifeste la présence latente du tueur

2. le temps en évolution lente des nappes aiguës, qui se meuvent au ralenti dans un continuum spatio-temporel et informent d'un déclenchement et d'une orientation du mouvement

3. le temps inexorablement « en marche » de l'évènement à venir. 


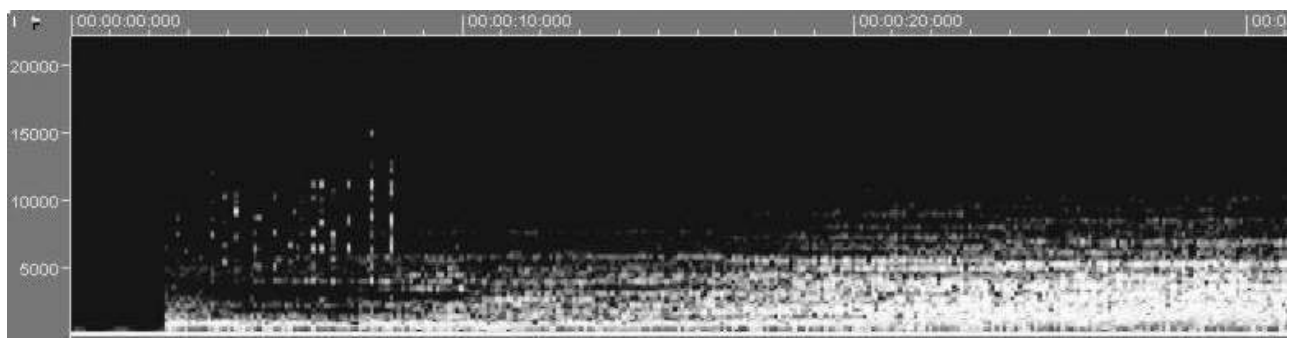

Cette deuxième pédale peut d'ailleurs, angoisse supplémentaire, devenir instable et comme «habitée » intérieurement par une trame micro-polyphonique -apparemmentaléatoire évoluant dans un ambitus restreint, créant ainsi une illusion de mobilité anarchique à l'intérieur même d'un espace voué par essence à l'immobilité, parfaite illustration d'un imaginaire du fourmillement: «le schème de l'animation accélérée qu'est l'agitation fourmillante, grouillante ou chaotique, semble être une projection assimilatrice de l'angoisse devant le changement (...). Or, le changement et l'adaptation qu'il motive est la première expérience du temps $»^{5}$, écrit Gilbert Durand.

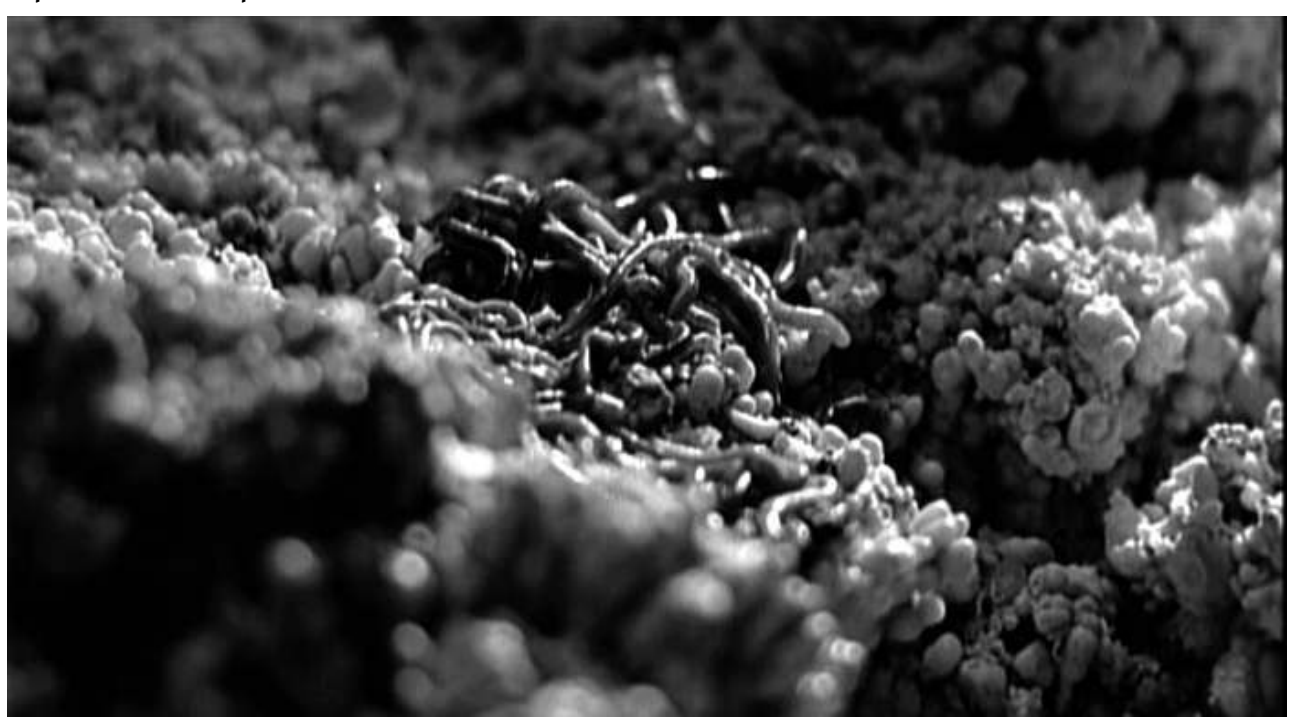

21 Tel est le cas dans Shining (réalisé par Stanley Kubrick, musiques de G. Ligeti, B. Bartok et Penderecki), où la musique qui accompagne la découverte par Wendy du «travail» d'écrivain de Jack, une phrase répétée à l'infini, superpose deux surfaces sonores situées respectivement aux deux extrémités de l'espace audible, l'une stable, l'autre instable. Le vide crée par la distension absolue de la texture, la zone de non-rencontre entre les nappes donne à ressentir l'absence marquée d'un centre de gravité de l'espace musical externe, absence vécue comme un manque auquel s'ajoute la tension née de la dialectique ordre/désordre. Ce défaut de point de repère résonne au sein de la séquence cinématographique comme une révélation de la folie de Jack, de sa raison perdue.

Il existe ainsi toutes sortes de déclinaisons possibles de l'utilisation de la note pédale : une note, d'abord longuement tenue, peut s'animer doucement, devenir rampante, évoluer dans un continuum de hauteurs, ou bien se segmenter en unités accentuées, ou encore jouer avec les dynamiques avec, par exemple, l'utilisation de crescendos de grande amplitude en un temps très court.

23 Dans Scanners (réalisé par David Cronenberg, musique de Howard Shore) au moment où Cameron Vale s'introduit dans l'usine Biocarbon Amalgamate, la pédale grave est à peine audible et n'a vocation qu'à colorer la texture sonore dont l'élément dynamisant est un 
glissando aérien ascendant et descendant contre-pointé par une note suraiguë. L'ensemble, d'une remarquable simplicité, voire nudité, renforce le caractère de "sobriété aliénante et déshumanisante » des décors et participe à la construction de la problématique centrale chez Cronenberg, à savoir l'orgueil démesuré du rationalisme. L'usine Biocarbon apparait comme un modèle d'organisation et de maitrise technique et cependant, la musique atteste de la duplicité fondamentale de cette apparence en nous donnant à entendre une polyphonie de matériaux élémentaires, définissant un très large espace, dans lequel les deux lignes supérieures et inférieures encadrent une courbe en mouvement d'aspect aléatoire (bruit du vent), figurant ainsi les limites hautes et basses qu'une entité « naturelle » cherche à explorer (ex. 7).

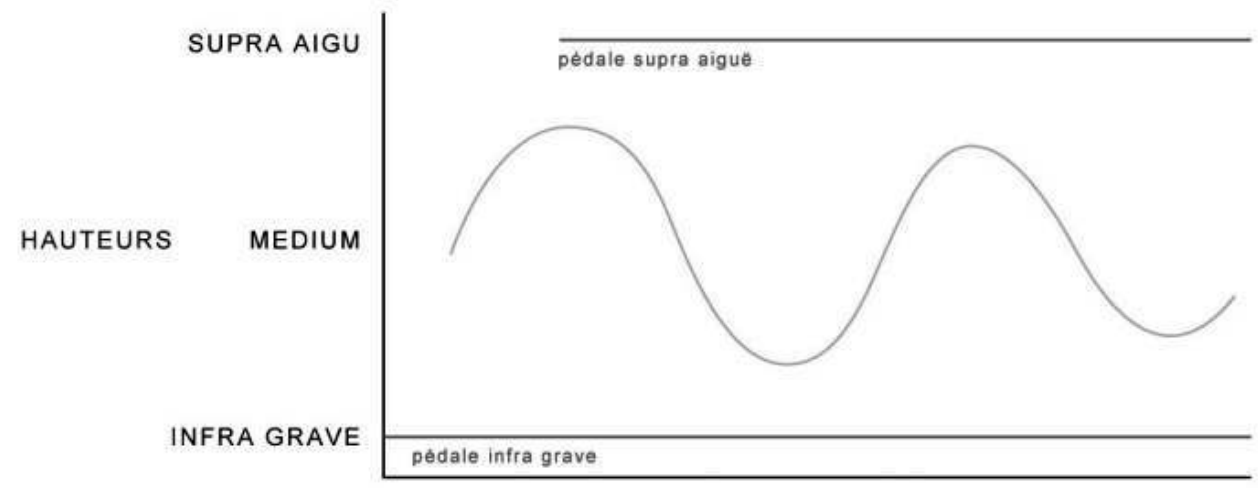

TEMPS

Si l'on considère les possibilités de symbolisation extrinsèques de la musique, l'ostinato et la note pédale sont bien les outils d'écriture privilégiés de l'effroi au cinéma. Le premier parce que sa nature même de formule rythmico-mélodique répétée obstinément est en rapport étroit avec le concept psychanalytique d'obsession qui «qualifie une idée, un sentiment ou une image qui s'impose de manière incoercible et répétée au sujet. Celui-ci (...) lutte en vain contre elle, et cette lutte s'accompagne d'angoisse $"^{6}:$ l'analogie entre état psychologique et le mouvement de l'énoncé musical semble donc pouvoir expliquer le mécanisme sémantique, d'autant plus que la musique est ici relayée par les images.

Le rôle de la pédale, dont les potentialités de renvois extra musicaux appartiennent davantage au champ spatial qu'au champ cinétique de l'ostinato, trouve une explication sensiblement identique, sauf qu'il ne s'agit plus alors d'analogie dans la catégorie de la temporalité, mais dans celle de la spatialité : l'obsession n'est plus liée à l'énonciation, mais à l'énoncé.

Ainsi, à la manière du madrigal de la renaissance et de l'opéra romantique, le cinéma d'horreur génère-t-il ses propres codes musicaux symboliques, en s'appuyant essentiellement sur des schèmes conventionnels certes, mais parfaitement adaptables aux objectifs recherchés. 


\section{NOTES}

1. Michelle Biget-Mainfroy, Le rêve et la fureur, Michel de Maule, Paris, 2002, p. 168.

2. Francis Decarsin, La musique, architecture du temps, l'Harmattan, Paris, 2001.

3. Ibid., p. 148.

4. Ibid., p. 152.

5. Gilbert Durand, Les structures anthropologiques de l'imaginaire, Dunod, Paris, 1992, p. 77.

6. Marie-Christine Hardy-Baylé, Le diagnostic en psychiatrie, Paris, Nathan, 1994, p. 66.

\section{AUTEUR}

\section{BERNARD ARBUS}

Pianiste. Professeur Agrégé de Musicologie à l'EREA de Berck 